\title{
Patient satisfaction with conventional dentures vs. digital dentures fabricated using 3D-printing: A randomized crossover trial
}

\author{
Katsura Ohara a,1, Yukari Isshiki ${ }^{a, 1},{ }^{*}$, Noriyuki Hoshi ${ }^{a}$, Akinori Ohno a , Norishige Kawanishi a, \\ Shintaro Nagashima a , Makoto Inoue a, Daijiro Kubo ${ }^{a}$, Katsuhiko Yamaya ${ }^{b}$, Erika Inoue ${ }^{b}$, \\ Katsuhiko Kimoto ${ }^{a}$ \\ a Department of Oral Interdisciplinary Medicine (Prosthodontics \& Oral Implantology), Graduate School of Dentistry, Kanagawa Dental University, Kanagawa, \\ Japan \\ b Technical Department of Kanagawa Dental University Hospital, Kanagawa, Japan
}

\begin{abstract}
Purpose: Evidence regarding the performance of digital dentures (DDs) fabricated using 3D printing is insufficient. This study aimed to evaluate patient satisfaction with conventional dentures (CDs) and DDs fabricated using 3D printing. Methods: This randomized controlled trial was conducted between November 2017 and May 2020. In the CD-DD group, CDs were fabricated before DDs, while the protocol was reversed in the DD-CD group. The primary outcome was patient satisfaction, quantified using a 100-mm visual analog scale, which assessed chewing efficiency, pain, stability, retention, comfort, esthetics, ease of cleaning, phonetics, and general satisfaction. Secondary outcomes were quality of life (QOL), number of visits, time required for definitive denture fabrication, number of adjustment appointments, and time required for denture stabilization after denture delivery.

Results: Patient satisfaction with CDs was superior in terms of phonetics, ease of cleaning, stability, comfort, and general satisfaction. Secondary outcomes such as social disability and the number of clinic visits were significantly lower in patients with DDs. However, the two groups showed no significant differences in the other outcomes. Although patient satisfaction with DDs was inferior to that with CDs, $20 \%$ of patients preferred and used DDs (12 patients preferred CDs, and three patients opted for DDs).

Conclusion: Although patient satisfaction or QOL with DDs may be somewhat inferior to that with CDs, $20 \%$ of patients preferred and used DDs daily. Thus, DDs fabricated using 3D printing may have comparable practicality and efficacy to CDs.
\end{abstract}

Keywords: Patient satisfaction, 3D-printed dentures, Crossover trial

Received 5 March 2021, Accepted 30 November 2021, Available online 27 January 2022

\section{Introduction}

In recent years, computer-aided design (CAD) and computeraided manufacturing (CAM) have been widely integrated into clinical dentistry, particularly in the field of prosthetics. Unlike conventional methods, the new workflows of these CAD/CAM systems enable the fabrication of high-performance restorations[1,2]. Duret et al., in 1988, first reported the use of CAD/CAM technology for prosthesis fabrication[3]. Since the 1980s, many researchers have focused on the practical applications of these systems[4-7]. The advantages of CAD/CAM systems include increased productivity, lower costs,

DOI: https://doi.org/10.2186/jpr.JPR_D_21_00048

${ }^{1}$ These authors contributed equally to this study.

*Corresponding author: Yukari Isshiki, Department of Oral Interdisciplinary Medicine (Prosthodontics \& Oral Implantology), Graduate School of Dentistry, Kanagawa Dental University, 82 Inaokacho, Yokosuka, Kanagawa 238-8580, Japan.

E-mail address: isshiki@kdu.ac.jp

Copyright: @ 2022 Japan Prosthodontic Society. All rights reserved. easier data management, and shorter treatment durations, which are beneficial for both dental surgeons and patients[8-10]. This technology has already been applied to fixed prosthetic appliances, such as inlays, crowns, and implant prosthetic components[11-14]. However, removable prostheses consist of several metallic (clasps, frameworks) and non-metallic (denture bases and artificial teeth) components, which render the application of the CAD/CAM system difficult. Therefore, the fabrication of removable prostheses using CAD/CAM has been delayed[15]. Currently, CAD/CAM complete dentures are fabricated by two companies (AvaDent; Global Dental Science, Scottsdale, AZ and DENTCA; Dentca Inc., Los Angeles, CA), and these dentures are available for use in edentulous patients[16-23]. Both companies allow definitive dentures to be fabricated in a few visits, and the dental materials, techniques, and fabrication processes have been established and standardized. The fabrication process for DENTCA dentures involves 3D printing technology (3DP) [24]. CAD/CAM dentures have been shown to achieve greater patient satisfaction than conventional complete dentures[19-22]. However, 
evidence regarding the performance of digital dentures (DDs) fabricated using 3DP is insufficient, and additional research data on this topic is required[23].

The disadvantages of milled dentures include the large size of production machines, low production efficiency, high time consumption, high cost, limited block size, and the need to discard cutting debris[25]. These disadvantages can be resolved if denture fabrication by 3DP becomes possible. Therefore, this randomized controlled trial aimed to compare patient satisfaction between conventional complete dentures and digital complete dentures fabricated using 3DP, which requires three patient visits in principle, including the try-in of trial dentures. This study also compared the patients' quality of life (QOL), number of visits, time required for definitive denture fabrication, number of adjustment appointments, and time required for denture stabilization after denture delivery as secondary outcomes. Although DDs can be fitted in three visits, there are limited reports describing the use of this system in Japan; therefore, we included this parameter in the survey to confirm whether DDs can actually be fitted in three visits. The null hypothesis was that there is no difference in patient satisfaction, $\mathrm{QOL}$, number of visits, and time required for definitive denture fabrication between DDs fabricated using 3DP and conventional dentures (CDs).

\section{Materials and Methods}

\subsection{Participants}

This study included edentulous men and women (aged 66-90 years). The inclusion criteria were as follows: (1) patients with edentulous upper and lower arches who visited our hospital for upper and lower complete denture fabrication; and (2) patients who had received sufficient explanation of the study, understood the procedures, and provided their written consent to participate in the study. The exclusion criteria were as follows: (1) patients with physical or mental disorders; (2) patients who could not understand, read, or write Japanese; and (3) patients with dietary restrictions.

This study was conducted after obtaining approval from the Research Ethics Review Committee of the authors' affiliated institution and was registered in the University Hospital Medical Information Network and the Japan Registry of Clinical Trials. All participants provided informed consent prior to participation in the study.

\subsection{Study Design}

A crossover randomized controlled trial was conducted between November 2017 and May 2020, in which a set of upper and lower CDs and a set of upper and lower DDs were fabricated using 3DP for each patient by three qualified prosthodontists.

Participants who wished to receive new complete dentures were continuously recruited and assigned to blocks by using a random number table by the principal investigator. Each block consisted of two groups: CD-DD and DD-CD. In the CD-DD group, the CDs were fabricated first, followed by the DDs, while the reverse order was followed in the DD-CD group (Fig. 1). The principal investigator managed the study but was not involved in the treatment.

The primary outcome was patient satisfaction assessed using a 100-mm visual analog scale (VAS)[26], which quantified chewing efficiency, pain, stability, retention, comfort, esthetics, ease of clean- ing, phonetics, and general satisfaction. The secondary outcomes were QOL assessed using the Japanese version of the Oral Health Impact Profile for edentulous patients (OHIP-EDENT-J)[27], number of visits, time required for definitive denture fabrication, number of adjustment visits, and time required for denture stabilization (until the patients were able to eat without pain after denture delivery). A stopwatch was used to measure the patients' treatment time (s), starting from when the patients sat down on the dental chair to when the patients stood up after the treatment. To avoid any bias in responses, the patients were told that the prosthodontist would not see their responses for the main evaluation. The questionnaire was completed by the patients in the absence of the prosthodontists. If the patients required any supplementary briefing regarding the questionnaire, the coordinator rather than the prosthodontist responded. Furthermore, the responses were sought at baseline and after denture adjustment. Since the CDs and DDs differed visually, blinding was not possible for both patients and prosthodontists. Blinding of the base material was deemed unfeasible because both the patients and prosthodontists were clearly aware of the type of material being used.

\subsection{Treatment Protocol}

In the fabrication of CDs, primary impressions were obtained using irreversible hydrocolloid impression material for the fabrication of individual trays. Border molding was performed using these individual trays and specific impression compounds (Iso Compound and Peri Compound, GC Corporation, Tokyo, Japan), and definitive impressions were obtained using vinyl polysiloxane impression material (Exafine regular type and injection type, GC Corporation, Tokyo, Japan). For the determination of denture occlusion, vertical and centric jaw relationships were recorded using record bases with wax occlusal rims. During the wax try-in, the occlusal relationship and esthetics of the wax trial dentures were verified. After denture processing, the definitive dentures were delivered to the patients and adjusted for any tissue impingement and/or occlusal interferences.

In the fabrication of DDs, the definitive impressions were recorded using DENTCA trays. After the try-in of the trays in the mouth, border molding was performed using a heavy-bodied vinyl polysiloxane impression material (Flexitime Heavy Trays; Kulzer Japan Co., Ltd., Tokyo, Japan), followed by definitive impressions using a light-bodied vinyl polysiloxane impression material (Flexitime Collect Flow, Ltd., Tokyo, Japan). After the vertical jaw relationship was recorded, the posterior border of the definitive impression was cut according to the posterior extent of the denture, gothic arch tracers were attached, and the centric position was determined on the same day. A vinyl polysiloxane bite material (Flexitime Bite; Kulzer Japan Co., Ltd., Tokyo, Japan) was used to record the intermaxillary relationship. A trial denture was fabricated using a 3D printer according to the definitive impressions and intermaxillary records, and the occlusion, vertical intermaxillary relationship, midline, occlusal plane, overjet, and overbite were evaluated. The DDs were delivered at the third clinic visit and were adjusted until they were as comfortable as the CDs (Fig. 2).

\subsection{Laboratory Protocol}

The laboratory process for CDs employed a plaster cast made from the primary impression, and individual trays were fabricated from polymethylmethacrylate (PMMA) resin. A definitive cast was poured from the definitive impression. The record base was waxed 


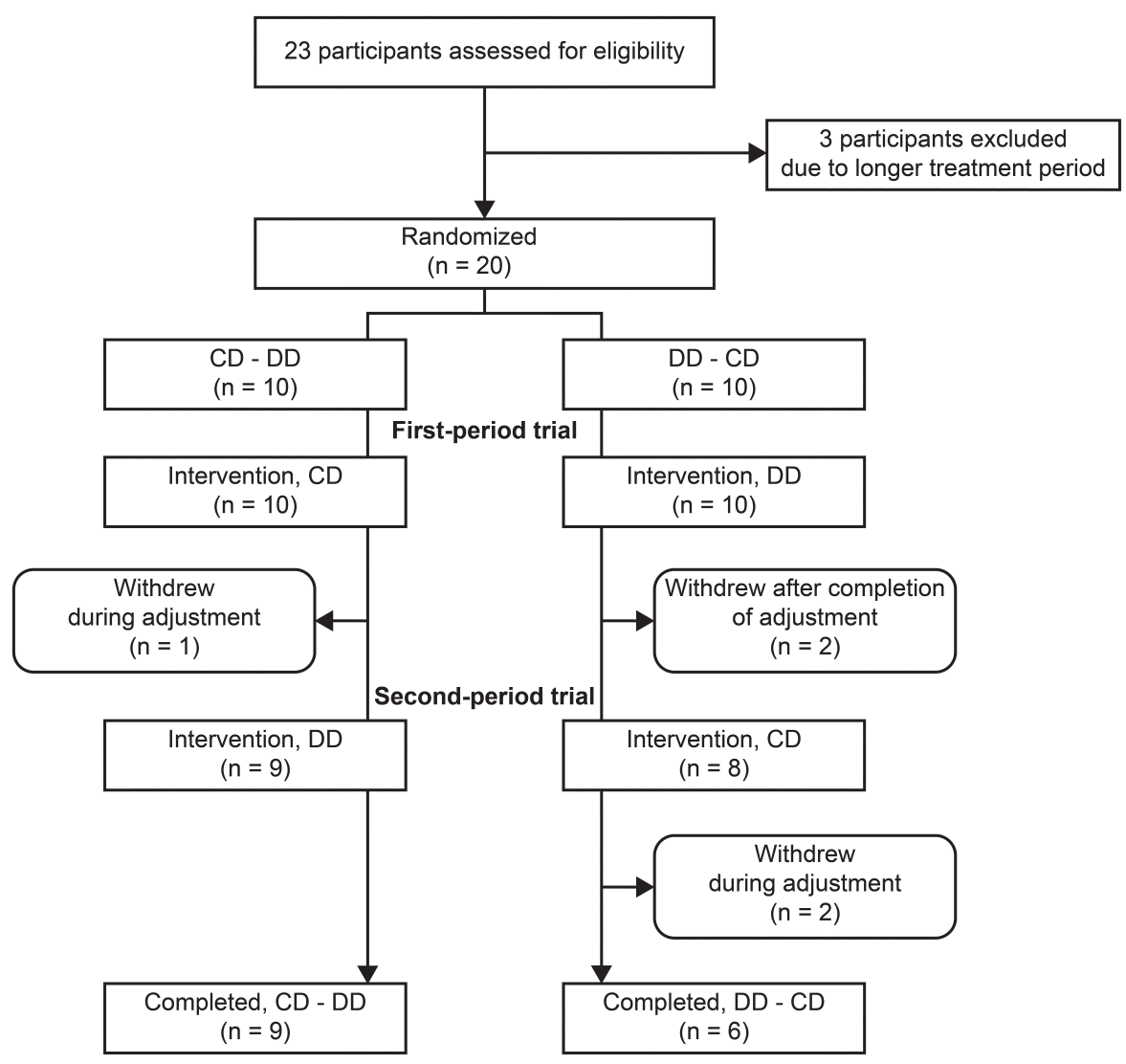

Fig. 1. Participant flow diagram.

to obtain wax occlusal rims. After recording the jaw relationships, artificial teeth (Verasia SA, SHOFU INC., Kyoto, Japan) were set in the occlusal rims, and the trial dentures were fabricated using heat-processed PMMA, trimmed, and polished by a prosthodontic technician. A single technician performed all laboratory procedures.

For the DDs, trial dentures (dima denture base try-in; Kulzer Japan Co., Ltd., Tokyo, Japan) were fabricated using a 3D printer (ZENiTH U; Yoshida Co., Ltd., Tokyo, Japan) based on data converted into digital files at the laboratory (Mitsui Chemicals, Inc.). In addition, the denture base (dima print denture base; Kulzer Japan Co., Ltd., Tokyo, Japan) and artificial teeth (dima print denture teeth; Kulzer Japan Co. Tokyo, Japan) were separately fabricated using a 3D printer[28-32].

\subsection{Statistical Analysis}

The sample size for this $2 \times 2$ crossover trial was calculated as follows: seeking a mean difference of $20 \mathrm{~mm}$ with a standard deviation of $30 \mathrm{~mm}$ in general satisfaction between the groups on the $100-\mathrm{mm}$ VAS[33]; 20 participants were required to obtain $80 \%$ power with a two-sided alpha level of $5 \%$.

Differences in baseline allocation and in the first and second dentures between the CD-DD and DD-CD groups were compared using the Mann-Whitney $U$ test. A generalized estimating equation was used to determine the relationship between CDs and DDs on the 100-mm VAS and the Japanese version of the Oral Health Impact Profile for Edentulous patients (OHIP-EDEN-J). A $t$-test was performed to determine whether the mean differences in the number and time of visits required for definitive denture fabrication and denture stabilization after denture delivery were statistically significant. Statistical analyses were conducted using SPSS statistical software (IBM Corporation, Armonk, NY). The significance level was set as $5 \%$.

\section{Results}

\subsection{Participants}

A total of 23 participants were screened, and 20 participants who provided informed consent were included. Three subjects did not provide consent because they were not interested in participating in a lengthy trial. There was no significant difference in the averages of the baseline characteristics between the CD-DD and DD-CD groups ( $p<0.05$ ) (Table 1). Of the 20 included subjects, five dropped out due to illness or hospitalization. In the CD-DD group, one participant was excluded because of hospitalization during the production of the first denture. In the DD-CD group, two participants were excluded after production of the first denture-one patient requested discontinuation from the trial because of an impending operation and the other patient was unable to visit the hospital due to health problems. Two additional participants were excluded after the production of the second denture; these two participants did not contact the investigators or attend any further visits. 
CD

\begin{tabular}{|c|}
\hline Preliminary impression \\
\hline $\begin{array}{c}\text { Irreversible hydrocolloid } \\
\text { impression in stock trays }\end{array}$ \\
\hline Definitive impression \\
\hline $\begin{array}{c}\text { Vinyl polysiloxane } \\
\text { impression in border-molded } \\
\text { individual trays }\end{array}$ \\
\hline $\begin{array}{c}\text { Maxillomandibular } \\
\text { relationship records }\end{array}$ \\
Try-in \\
\hline
\end{tabular}

Fig. 2. Comparison of conventional and digital clinical workflows.

\subsection{Outcomes}

The VAS and OHIP scores did not differ significantly between the first and second set of dentures in the patient groups. With regard to patient satisfaction, which was quantified by VAS scores, CDs scored significantly higher than DDs in terms of phonetics, ease of cleaning, stability, comfort, and general satisfaction (Table 2). In contrast, on the OHIP-EDENT-J, DDs scored significantly higher in social disability $(p<0.05)$ but showed no significant differences in other areas (Table 3). After the evaluation, the participants were asked to select either the CD or DD; 12 participants selected CDs and three selected DDs (Fig. 3).

The number of visits required for definitive denture fabrication, including the number of remakes, was significantly lower for DDs (Fig. 4). However, there were no significant differences in the number of adjustment sessions and the time required for denture fabrication and denture adjustment between CDs and DDs (Figs. 4 and 5).

\section{Discussion}

This study aimed to compare patient satisfaction between conventional complete dentures and digital complete dentures fabricated using 3DP. Various benefits of DDs have previously been reported, such as improved surgeon/patient satisfaction, reduced number of visits and chair time, improved denture fit, improved ease of maintenance, greater consistency and durability of the prosthodontic appliances, ability to store digital information, reduced workload for technicians, and shorter fabrication time[34-38]. However, our results revealed that CDs were significantly better than DDs in terms of several assessment items related to patient satisfaction, in contrast to earlier case reports[21,22]. We believe that the primary reason for this result could be the difference in the manufacturing methods be-
Table 1. Baseline characteristics of the 20 subjects enrolled in the trial

\begin{tabular}{|c|c|c|c|c|}
\hline & \multicolumn{2}{|c|}{ Group } & \multirow{2}{*}{$\begin{array}{l}\text { Total } \\
(n=20)\end{array}$} & \multirow[t]{2}{*}{$p$-value } \\
\hline & $\begin{array}{l}\mathrm{DD}-\mathrm{CD} \\
(\mathrm{n}=10)\end{array}$ & $\begin{array}{l}C D-D D \\
(n=10)\end{array}$ & & \\
\hline Age (y) & $85[79,89]$ & $77[70,87]$ & $82[74,88]$ & $.739^{a}$ \\
\hline $\begin{array}{l}\text { Gender } \\
(\%)\end{array}$ & & & & $.653^{b}$ \\
\hline Female & $5(50 \%)$ & $4(40 \%)$ & $9(45 \%)$ & \\
\hline Male & $5(50 \%)$ & $6(60 \%)$ & $11(55 \%)$ & \\
\hline Edentulous Period (y) & $12[9,21]$ & $7[1,31]$ & $10[2,27]$ & $.529^{\mathrm{a}}$ \\
\hline Age of previous dentures (y) & $3[0,5]$ & $2[1,6]$ & $2[1,6]$ & $.089^{\mathrm{a}}$ \\
\hline Number of previous dentures & $2[2,4]$ & $1[1,2]$ & $2[1,3]$ & $.631^{\mathrm{a}}$ \\
\hline $\begin{array}{l}\text { American College of } \\
\text { Prosthodontists' classification (\%) }\end{array}$ & & & & $.684^{b}$ \\
\hline । & $1(10 \%)$ & $2(20 \%)$ & $3(15 \%)$ & \\
\hline ॥ & $3(30 \%)$ & $4(40 \%)$ & $7(35 \%)$ & \\
\hline III & $4(40 \%)$ & $2(20 \%)$ & $6(30 \%)$ & \\
\hline IV & $2(20 \%)$ & $2(20 \%)$ & $4(20 \%)$ & \\
\hline $\begin{array}{l}\text { Classification (Japan } \\
\text { Prosthodontic Society) }\end{array}$ & & & & $.529^{b}$ \\
\hline I & $3(30 \%)$ & $4(40 \%)$ & $7(35 \%)$ & \\
\hline ॥ & $3(30 \%)$ & $2(20 \%)$ & $5(25 \%)$ & \\
\hline III & $3(30 \%)$ & $3(30 \%)$ & $6(30 \%)$ & \\
\hline IV & $1(10 \%)$ & $1(10 \%)$ & $2(10 \%)$ & \\
\hline
\end{tabular}

tween DDs fabricated using a milling machine and those fabricated using a 3D printer. Since dentures fabricated using milling machines are milled from resin blocks, their physical properties and suitability have been reported to be equal to or better than those of CDs and 3DP-fabricated dentures[39,40].

Furthermore, 3DP dentures are currently fabricated with a palatal thickness of $2.5 \mathrm{~mm}$ to ensure strength, while milled dentures and CDs are fabricated with a thickness that is approximately equivalent to that of a paraffin wax sheet (approximately $1.4 \mathrm{~mm}$ ). This is reflected in the significantly higher level of satisfaction in phonetics with CDs than with DDs. Additionally, the significantly higher satisfaction in ease of cleaning CDs compared to DDs may be attributed to the difference in the type of artificial teeth used. The artificial teeth (Verasia SA, SHOFU INC., Kyoto, Japan) used in milled dentures and $\mathrm{CDs}$ are manufactured from hard resin that exhibits color gradation. These artificial teeth have greater visual similarity to real teeth, whereas 3DP artificial teeth (dima print denture teeth) are similar to resin teeth in terms of physical properties but have a single-color tone. Moreover, since anterior artificial teeth designed by CAD consist of a single component with six teeth[32], it is difficult to create an uneven profile or a profile with a different axis for each tooth, which is possible with CDs. Although the dima print denture teeth in DDs have physical properties similar to those of resin teeth[28], DDs may become discolored more readily than $\mathrm{CDs}$. This may also be a primary factor contributing to our results.

The higher satisfaction with CDs in terms of stability and comfort may be attributed to the marginal seal at the denture borders and stable occlusal contacts. DDs are designed to incorporate the 
Table 2. Mean (standard error), generalized estimating equations, and $p$ values of the satisfaction ratings for the conventional and digital dentures on the $100-\mathrm{mm}$ visual analog scale

\begin{tabular}{llcccc}
\hline Parameter & \multicolumn{2}{c}{$\begin{array}{c}\text { CD } \\
\text { Mean }\end{array}$} & \multicolumn{2}{c}{$\begin{array}{c}\text { DD } \\
\text { (standard error) }\end{array}$} & p-value \\
& 73.89 & $(6.47)$ & 60.66 & $(5.80)$ & \\
\hline Chewing efficiency & 83.80 & $(5.64)$ & 69.08 & $(5.05)$ & $0.005^{*}$ \\
Phonetics & 92.93 & $(3.44)$ & 86.20 & $(3.16)$ & $0.001^{*}$ \\
Ease of cleaning & 75.87 & $(5.11)$ & 68.46 & $(4.92)$ & $0.012^{*}$ \\
Stability & 78.11 & $(6.08)$ & 63.50 & $(5.46)$ & \\
Retention & 78.24 & $(5.04)$ & 62.88 & $(4.79)$ & $0.004^{*}$ \\
Comfort & 87.55 & $(5.60)$ & 77.67 & $(5.22)$ & \\
Esthetics & 76.66 & $(5.82)$ & 81.09 & $(5.25)$ & \\
Pain & 78.83 & $(6.41)$ & 61.10 & $(5.75)$ & $0.016^{*}$ \\
General satisfaction & &
\end{tabular}

$\mathrm{CD}$, Conventional dentures; DD, Digital dentures

*statistically significant

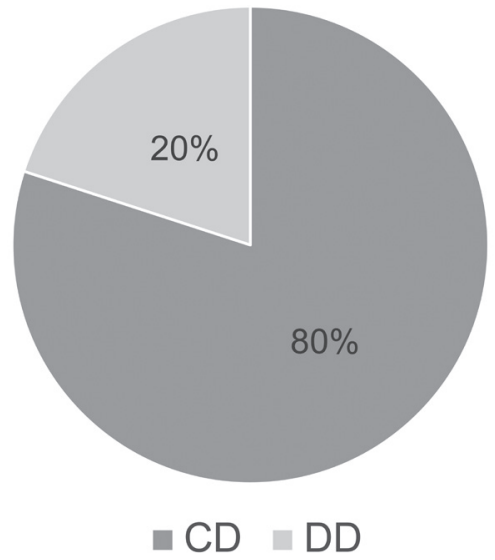

Fig. 3. Denture preference.

maximally extended impression, and the thickness of the fringe is determined arbitrarily; thus, the marginal seal of DDs may be inferior to that of CDs. Jo et al. reported that when a skilled dentist fabricates complete dentures for an edentulous mandible, patient satisfaction is higher with the conventional method than with the simplified method, wherein no definitive impressions are recorded[41]. In this study, satisfaction with CDs may have been higher because the dentures were fabricated by an experienced prosthodontist.

DD is expected to reduce the physical burden on patients and increase the utility of home visits by reducing the number of visits to the clinic required for denture fabrication[19,23]. However, regrettably, we were unable to identify any improvement in the time required for denture fabrication. Several prior reports have highlighted the procedural errors in recording occlusion with DDs[16,19,42,43], and these factors may have contributed to the longer treatment time.

This study had several limitations. First, the washout period was not long enough. Implementation of a formal washout period was not ethically feasible in this study, since it would have necessitated a period of time when the denture was not used. However, as shown in the results, the influence of the carryover effect can be considered to be small, because there was no significant difference noted in the
Table 3. Mean (standard error), generalized estimating equations, and $p$-values of the Japanese version of the Oral Health Impact Profile for edentulous patients for conventional and digital dentures

\begin{tabular}{|c|c|c|c|c|c|}
\hline \multirow{2}{*}{$\begin{array}{l}\text { Parameter } \\
\text { Functional } \\
\text { Limitation }\end{array}$} & \multicolumn{2}{|c|}{$\begin{array}{c}\text { CD } \\
\text { Mean } \\
\text { (standard error) }\end{array}$} & \multicolumn{2}{|c|}{$\begin{array}{c}\text { DD } \\
\text { Mean } \\
\text { (standard error) }\end{array}$} & \multirow{2}{*}{$\frac{p \text {-value }}{0.318}$} \\
\hline & 1.425 & (0.199) & 1.549 & $(0.181)$ & \\
\hline Physical pain & 1.349 & $(0.207)$ & 1.462 & (0.193) & \\
\hline $\begin{array}{l}\text { Psychological } \\
\text { discomfort }\end{array}$ & 1.173 & $(0.246)$ & 1.395 & $(0.222)$ & \\
\hline $\begin{array}{l}\text { Physical } \\
\text { disability }\end{array}$ & 1.346 & $(0.244)$ & 1.676 & $(0.222)$ & \\
\hline $\begin{array}{l}\text { Psychological } \\
\text { disability }\end{array}$ & 0.438 & $(0.202)$ & 0.602 & $(0.181)$ & 0.480 \\
\hline Social disability & 0.150 & $(0.179)$ & 0.690 & $(0.161)$ & $0.001^{*}$ \\
\hline Handicap & 0.563 & $(0.245)$ & 0.789 & $(0.226)$ & \\
\hline
\end{tabular}

CD, Conventional dentures; DD, Digital dentures

${ }^{*}$ statistically significant

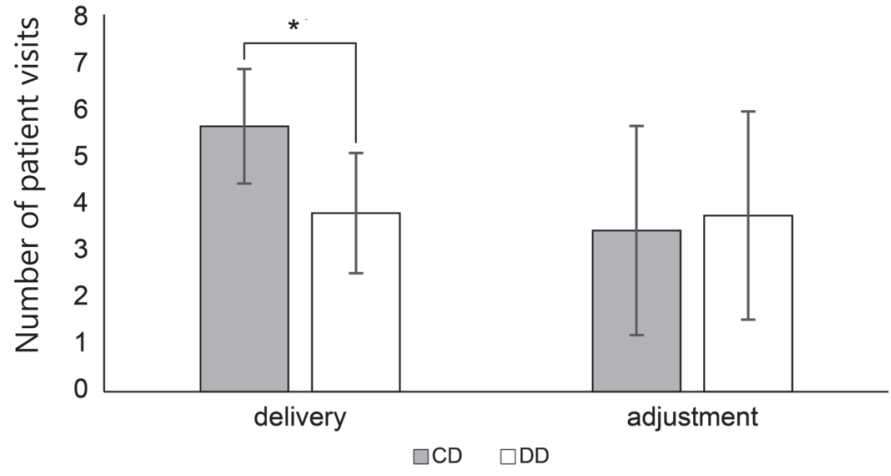

Fig. 4. Number of patient visits required for denture fabrication and adjustment.

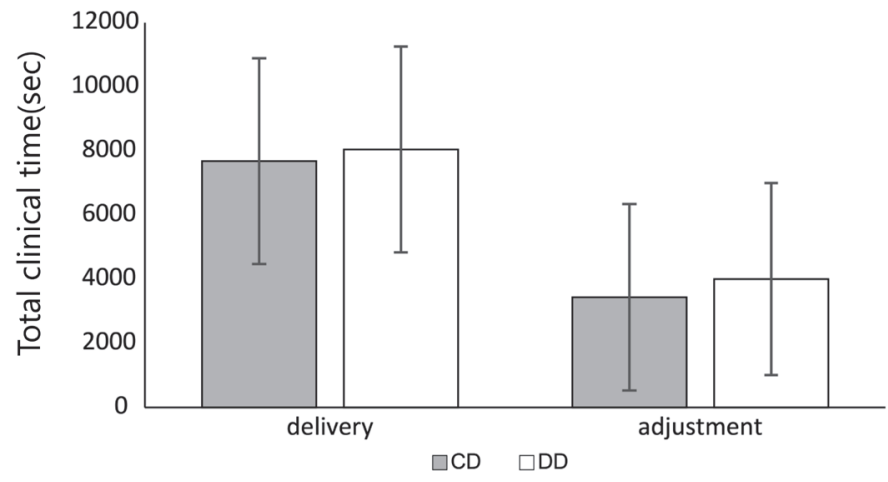

Fig. 5. Total clinical time required for denture fabrication and adjustment.

VAS and OHIP between the first and second set of dentures in the patient groups $(p<0.05)$.

Second, we could not evaluate the medium- and long-term prognosis of DD since $80 \%$ of the patients chose CD, which is also a limitation of the crossover design. This problem can be solved by developing a new parallel research design in the future. Moreover, 
since this study was conducted in elderly patients, the dropout rate was higher than expected, indicating the need for a larger sample size.

The current 3DP-based DD system is still in the developmental stage and shows numerous potential problems, including procedural errors, conformity with mucosal surfaces, stable occlusion, dimensional changes due to post-processing[44], and deterioration of physical properties and esthetics. Further improvements and refinements are necessary to enable its use in routine clinical applications.

\section{Conclusion}

Within the limitations of this crossover randomized controlled study, the following conclusions can be drawn:

1. Patient satisfaction with CDs was superior than that with DDs in terms of phonetics, ease of cleaning, stability, comfort, and general satisfaction.

2. With regard to patients' QOL in the context of social disability, CDs were superior to DDs.

3. Although DD involved fewer clinic visits, the time required for denture fabrication, number of adjustments, and the time required for adjustments did not differ between DDs and CDs.

4. The clinical trial findings suggested that $20 \%$ of patients preferred and used DDs daily. Thus, DDs fabricated using 3D printing may be comparable to CDs in terms of practicality and efficacy.

\section{Acknowledgements}

We would like to express our sincere gratitude to the study participants, faculty of the Department of Oral and Integrative Medicine of our university for their support and cooperation, and Professor Ayumi Shintani of the Department of Medical Statistics, Graduate School of Medicine, Osaka City University, for her guidance in statistical analysis.

This study was supported by the Ministry of Education, Culture, Sports, Science and Technology of Japan (MEXT) 2019 Grant-in-Aid for Young Scientists (No. 19K19144). The funding agency had no role in the study design; collection, analysis, and interpretation of data; writing of the manuscript; and decision to submit the manuscript for publication.

\section{Conflicts of interest}

The authors have no conflict of interest to declare.

\section{References}

[1] Ahmed KE. We're going digital: the current state of CAD/CAM dentistry in prosthodontics. Prim Dent J. 2018;7:30-5. https://doi. org/10.1177/205016841800700205, PMID:30095879

[2] Kattadiyil MT, AlHelal A. An update on computer-engineered complete dentures: A systematic review on clinical outcomes. J Prosthet Dent. 2017;117:478-85. https://doi.org/10.1016/j.prosdent.2016.08.017, PMID:27881317

[3] Duret F, Blouin JL, Duret B. CAD-CAM in dentistry. J Am Dent Assoc. 1988;117:715-20. https://doi.org/10.14219/jada.archive.1988.0096, PMID:3058771

[4] Mörmann WH, Brandestini M, Lutz F, Barbakow F. Chairside computer-aided direct ceramic inlays. Quintessence Int. 1989;20:329-39. PMID:2756089

[5] Rekow D. Computer-aided design and manufacturing in dentistry: A review of the state of the art. J Prosthet Dent. 1987;58:512-6. https://doi. org/10.1016/0022-3913(87)90285-X, PMID:3312586
[6] Fujita T. Preliminary report on construction of prosthetic restorations by means of computer aided design (CAD) and numerically controlled (NC) machine tools. Bull Kanagawa Dent Coll. 1984;12:75-7.

[7] Miyazaki T, Hotta Y, Kunii J, Kuriyama S, Tamaki Y. A review of dental CAD/ CAM: current status and future perspectives from 20 years of experience. Dent Mater J. 2009;28:44-56. https://doi.org/10.4012/dmj.28.44, PMID:19280967

[8] Beuer F, Schweiger J, Edelhoff D. Digital dentistry: an overview of recent developments for CAD/CAM generated restorations. Br Dent J. 2008;204:50511. https://doi.org/10.1038/sj.bdj.2008.350, PMID:18469768

[9] van Noort R. The future of dental devices is digital. Dent Mater. 2012;28:3-12. https://doi.org/10.1016/j.dental.2011.10.014, PMID:22119539

[10] Blatz MB, Vonderheide $M$, Conejo J. The effect of resin bonding on longterm success of high-strength ceramics. J Dent Res. 2018;97:132-9. https:// doi.org/10.1177/0022034517729134, PMID:28876966

[11] Mello C, Lemos C, Gomes J, Verri F, Pellizzer E. CAD/CAM vs conventional technique for fabrication of implant-supported frameworks: A systematic review and meta-analysis of in vitro studies. Int J Prosthodont. 2019;32:18292. https://doi.org/10.11607/ijp.5616, PMID:30856643

[12] Spitznagel FA, Boldt J, Gierthmuehlen PC. CAD/CAM ceramic restorative materials for natural teeth. J Dent Res. 2018;97:1082-91. https://doi. org/10.1177/0022034518779759, PMID:29906206

[13] Papadiochou S, Pissiotis AL. Marginal adaptation and CAD-CAM technology: A systematic review of restorative material and fabrication techniques. J Prosthet Dent. 2018;119:545-51. https://doi.org/10.1016/j. prosdent.2017.07.001, PMID:28967399

[14] Carneiro Pereira AL, Bezerra de Medeiros AK, de Sousa Santos K, Oliveira de Almeida É, Seabra Barbosa GA, da Fonte Porto Carreiro A. Accuracy of CADCAM systems for removable partial denture framework fabrication: A systematic review. J Prosthet Dent. 2021;125:241-8. https://doi.org/10.1016/j. prosdent.2020.01.003, PMID:32147252

[15] Wang C, Shi YF, Xie PJ, Wu JH. Accuracy of digital complete dentures: A systematic review of in vitro studies. J Prosthet Dent. 2021;125:249-56. https:// doi.org/10.1016/j.prosdent.2020.01.004, PMID:32115218

[16] Kattadiyil MT, GoodacreCJ, Baba NZ.CAD/CAM complete dentures: a review of two commercial fabrication systems. J Calif Dent Assoc. 2013;41:407-16. PMID:23875432

[17] Goodacre CJ, Garbacea A, Naylor WP, Daher T, Marchack CB, Lowry J. CAD/ CAM fabricated complete dentures: concepts and clinical methods of obtaining required morphological data. J Prosthet Dent. 2012;107:34-46. https://doi.org/10.1016/S0022-3913(12)60015-8, PMID:22230914

[18] Infante L, Yilmaz B, McGlumphy E, Finger I. Fabricating complete dentures with CAD/CAM technology. J Prosthet Dent. 2014;111:351-5. https://doi. org/10.1016/j.prosdent.2013.10.014, PMID:24461946

[19] Bidra AS, Farrell K, Burnham D, Dhingra A, Taylor TD, Kuo CL. Prospective cohort pilot study of 2-visit CAD/CAM monolithic complete dentures and implant-retained overdentures: clinical and patient-centered outcomes. J Prosthet Dent. 2016;115:578-586.e1. https://doi.org/10.1016/j.prosdent.2015.10.023, PMID:26794695

[20] Saponaro PC, Yilmaz B, Johnston W, Heshmati RH, McGlumphy EA. Evaluation of patient experience and satisfaction with CAD-CAM-fabricated complete dentures: A retrospective survey study. J Prosthet Dent. 2016;116:5248. https://doi.org/10.1016/j.prosdent.2016.01.034, PMID:27402416

[21] Jurado C, Tsujimoto A, Alhotan A, Villalobos-Tinoco J, AlShabib A. Digitally fabricated immediate complete dentures: case reports of milled and printed dentures. Int J Prosthodont. 2020;33:232-41. https://doi.org/10.11607/ ijp.6305, PMID:32069350

[22] Pereyra NM, Marano J, Subramanian G, Quek S, Leff D. Comparison of patient satisfaction in the fabrication of conventional dentures vs. DENTCA (CAD/CAM) dentures: A case report. J N J Dent Assoc. 2015;86:26-33. PMID:26242110

[23] Bidra AS, Taylor TD, Agar JR. Computer-aided technology for fabricating complete dentures: systematic review of historical background, current status, and future perspectives. J Prosthet Dent. 2013;109:361-6. https:// doi.org/10.1016/S0022-3913(13)60318-2, PMID:23763779

[24] Tae HK, Fabiana V. 3D printed complete dentures. Quintessence Dent Technol. 2016;141-9.

[25] Park ME, Shin SY. Three-dimensional comparative study on the accuracy and reproducibility of dental casts fabricated by 3D printers. J Prosthet Dent. 2018;119:861.e1-7. https://doi.org/10.1016/j.prosdent.2017.08.020, PMID:29475753 
[26] Awad MA, Feine JS. Measuring patient satisfaction with mandibular prostheses. Community Dent Oral Epidemiol. 1998;26:400-5. https://doi. org/10.1111/j.1600-0528.1998.tb01978.x, PMID:9870539

[27] Sato Y, Kaiba Y, Yamaga E, Minakuchi S. Reliability and validity of a Japanese version of the Oral Health Impact Profile for edentulous subjects. Gerodontology. 2012;29:1033-e1037. https://doi.org/10.1111/j.17412358.2011.00606.x, PMID:22187955

[28] Cha HS, Park JM, Kim TH, Lee JH. Wear resistance of 3D-printed denture tooth resin opposing zirconia and metal antagonists. J Prosthet Dent. 2020;124:387-94. https://doi.org/10.1016/j.prosdent.2019.09.004, PMID:31784192

[29] Chung YJ, Park JM, Kim TH, Ahn JS, Cha HS, Lee JH. 3D printing of resin material for denture artificial teeth: chipping and indirect tensile fracture resistance. Materials (Basel). 2018;11:1798. https://doi.org/10.3390/ma11101798, PMID:30248955

[30] Ahmed HA, Jose NJL. Available CAD/CAM system concepts for the fabrication of digital dentures. J Am Sci. 2019;15:47-55.

[31] Lee D-H, Lee J-S. Comparison of flexural strength according to thickness between CAD/CAM denture base resins and conventional denture base resins. J Dent Rehabil Appl Sci. 2020;36:183-95. https://doi.org/10.14368/ jdras.2020.36.3.183

[32] Dentca CAD/CAM denture. Clinical/product line knowledge base, https:// www.oralartsdental.com/wp-content/uploads/Dentca-3D-PrintableClinical-handout.pdf; 2017 [accessed 15 Jan 2021].

[33] Kimoto S, Kimoto K, Murakami H, Atsuko G, Ogawa A, Kawai Y. Effect of an acrylic resin-based resilient liner applied to mandibular complete dentures on satisfaction ratings among edentulous patients. Int J Prosthodont. 2014;27:561-6. https://doi.org/10.11607/ijp.3935, PMID:25390871

[34] AlHelal A, AIRumaih HS, Kattadiyil MT, Baba NZ, Goodacre CJ. Comparison of retention between maxillary milled and conventional denture bases: A clinical study. J Prosthet Dent. 2017;117:233-8. https://doi.org/10.1016/j. prosdent.2016.08.007, PMID:27765399

[35] Steinmassl PA, Klaunzer F, Steinmassl O, Dumfahrt H, Grunert I. Evaluation of currently available CAD/CAM denture systems. Int J Prosthodont. 2017;30:116-22. https://doi.org/10.11607/ijp.5031, PMID:28267817
[36] Janeva NM, Kovacevska G, Elencevski S, Panchevska S, Mijoska A, Lazarevska $B$. Advantages of CAD/CAM versus conventional complete dentures - A review. Open Access Maced J Med Sci. 2018;6:1498-502. https://doi. org/10.3889/oamjms.2018.308, PMID:30159084

[37] Bilgin MS, Erdem A, Aglarci OS, Dilber E. Fabricating complete dentures with CAD/CAM and RP technologies. J Prosthodont. 2015;24:576-9. https:// doi.org/10.1111/jopr.12302, PMID:26032438

[38] Kattadiyil MT, Jekki R, Goodacre CJ, Baba NZ. Comparison of treatment outcomes in digital and conventional complete removable dental prosthesis fabrications in a predoctoral setting. J Prosthet Dent. 2015;114:818-25. https://doi.org/10.1016/j.prosdent.2015.08.001, PMID:26412000

[39] Srinivasan M, Gjengedal H, Cattani-Lorente M, Moussa M, Durual S, Schimmel $M$, et al. CAD/CAM milled complete removable dental prostheses: An $<i>$ in vitro $</ \mathrm{i}>$ evaluation of biocompatibility, mechanical properties, and surface roughness. Dent Mater J. 2018;37:526-33. https://doi.org/10.4012/ dmj.2017-207, PMID:29515054

[40] Kalberer N, Mehl A, Schimmel M, Müller F, Srinivasan M. CAD-CAM milled versus rapidly prototyped (3D-printed) complete dentures: an in vitro evaluation of trueness. J Prosthet Dent. 2019;121:637-43. https://doi. org/10.1016/j.prosdent.2018.09.001, PMID:30711292

[41] Jo A, Kanazawa M, Sato Y, Iwaki M, Akiba N, Minakuchi S. A randomized controlled trial of the different impression methods for the complete denture fabrication: patient reported outcomes. J Dent. 2015;43:989-96. https://doi. org/10.1016/j.jdent.2015.05.007, PMID:26051546

[42] Kanazawa M, Inokoshi M, Minakuchi S, Ohbayashi N. Trial of a CAD/CAM system for fabricating complete dentures. Dent Mater J. 2011;30:93-6. https://doi.org/10.4012/dmj.2010-112, PMID:21282882

[43] Saponaro PC, Yilmaz B, Heshmati RH, McGlumphy EA. Clinical performance of CAD-CAM-fabricated complete dentures: A cross-sectional study. J Prosthet Dent. 2016;116:431-5. https://doi.org/10.1016/j.prosdent.2016.03.017, PMID:27160780

[44] Yoshitome K, Shimpo H, Kohgo O, Okubo C. Influence of building direction of additive manufacturing on fitting accuracy of 3D printed complete denture. Journal of the Japan Academy of Digital Dentistry. 2019;9:118.

This is an open-access article distributed under the terms of Creative Commons Attribution-NonCommercial License 4.0 (CC BYNC 4.0), which allows users to distribute and copy the material in any format as long as credit is given to the Japan Prosthodontic Society. It should be noted however, that the material cannot be used for commercial purposes. 